\title{
COMMUNICATION
}

\section{Un cas de lésion du nerf alvéolaire inférieur ayant contribué à déterminer l'apparition d'un trouble du comportement alimentaire.}

\author{
Dessalces Olenisac C1, Fauroux MA1 1 , Torres JH
}

1. Service d'Odontologie de Montpellier - Université Montpellier I : EA2415

\section{Introduction}

Les troubles du comportement alimentaire (TCA) prennent une ampleur croissante, tant au plan épidémiologique qu'au plan médiatique. En grande majorité, ils concernent des adolescents en majorité des jeunes femmes mais aussi de plus en plus de jeunes hommes, qui vénèrent le culte de la minceur (1), ayant recours à des moyens extrêmes pour atteindre leur idéal et sans conscience de la dangerosité des TCA. A première vue, une relation entre soins dentaires et aggravation d'une pathologie psychiatrique telle qu'un TCA parait peu probable. Cependant, cette relation a pourtant déjà été évoquée lors de l'introduction de matériel d'orthodontie dans la bouche, ou la pratique de certains actes de chirurgie orale qui auraient eu comme conséquence d'exacerber le TCA chez des femmes anorexiques ou boulimiques, voire de les faire rechuter dans leur pathologie (2) . L'explication fournie par la majorité des patientes interrogés serait que les douleurs postopératoires leur auraient fourni une justification à ne pas manger, ou leur auraient permis de perdre du poids, déclenchant ainsi une satisfaction exacerbant leur TCA.

\section{Observation}

Lors d'une visite au sein du service des troubles métaboliques du $\mathrm{CHU}$, une rencontre a été faite avec une patiente de 33 ans atteinte d'anorexie mentale restrictive depuis 5 ans. Elle pesait $22,6 \mathrm{Kg}$ pour $150 \mathrm{~cm}$, soit un indice de masse corporelle (IMC) de 10,1 (la norme étant située entre 18,5 et $25 \mathrm{~kg} / \mathrm{cm}^{2}$ d'après l'HAS). Cette patiente a déclaré être entrée dans la maladie à la suite de l'avulsion de la dent de sagesse 48. Lors de cette intervention, le nerf alvéolaire inférieur droit a été lésé, ce qui a entraîné une anesthésie labiomentonnière complète. Selon elle, le territoire concerné par ce trouble sensitif avait diminué en taille, mais une zone d'anesthésie complète persistait lors de notre examen.

\section{Discussion}

On ne peut pas conclure que l'acte chirurgical a pu constituer à lui seul le facteur étio-pathogénique déterminant l'apparition du TCA. Cependant, combiné à un contexte psychiatrique favorable, il a vraisemblablement pu actionner, chez cette patiente, le processus de restriction alimentaire.

\section{Conclusion}

Si des douleurs postopératoires ordinaires peuvent aggraver un TCA, une complication telle que la lésion du nerf alvéolaire inférieur semble à plus forte raison susceptible de constituer un facteur déclenchant et non plus seulement aggravant d'un TCA.

dessalces.christel@gmail.com

\section{Références}

(1) Jeammet P Anorexie, boulimie : les paradoxes de l'adolescence, Paris, Hachette littératures, $2004: 122-200$.

(2) Maine M et al. The role of third molar surgery in the exacerbation of eating disorders. J. Oral Maxillofac. Surg. 2001;59:1297-300.

(C) The authors, published by EDP Sciences. This is an Open Access article distributed under the terms of the Creative Commons Attribution License 4.0 (http://creativecommons.org/licenses/by/4.0/). 